\title{
Modelling of Profile Control with Lower Hybrid Wave Injection in the HL-2A Single-Null Divertor Plasma
}

\author{
Q.D. Gao*, H. Wang and B.S. Yuan
}

Southwestern Institute of Physics, P O Box 432, Chengdu 610041, P.R. China

\begin{abstract}
Separatrix of the single-null diverted plasma produced in the HL-2A tokamak is determined by a fast algorithm for identifying plasma boundary. Higher power LH wave $(1.5 \mathrm{MW})$ is injected into the diverted plasma with a slightly asymmetric spectrum. Plasma heating by electron Landau interaction results in operation scenarios of preferentially dominant electron heating. Current profile control under the dominant electron heating condition is investigated with numerical simulation. Due to the off-axis driven current, an RS q-profile is formed, and an enhanced confinement regime with steep electron temperature gradient is produced. The clear decrease of the electron thermal conductivity in the vicinity of the shear reversal shows that an electron-ITB is developed. When higher LH power injects into the target plasma that is heated by neutral beam injection, the ion temperature has a large increment in addition to the high increase of electron temperature. The temperature profiles indicate that an enhanced core confinement is established with both ionITB and electron-ITB developed.
\end{abstract}

\section{INTRODUCTION}

In the HL-2A tokamak (plasma major radius $\mathrm{R}=1.65 \mathrm{~m}$, minor radius $\mathrm{a}=0.4 \mathrm{~m}$, magnetic field at the center $\mathrm{B}_{\mathrm{T}}=$ $2.8 \mathrm{~T}$, and plasma current $\mathrm{I}_{\mathrm{p}}=0.48 \mathrm{MA}$ ), a single-null divertor (SND) configuration has been established in Ohmic discharges. In order to raise the plasma parameters and achieve more interesting operation scenarios in HL-2A [1], a campaign to carry out plasma heating using the available auxiliary heating schemes is essential. Now the auxiliary heating scheme of lower hybrid (LH) wave (2 klystrons with power up to $2.0 \mathrm{MW}$ at frequency $\mathrm{f}=2.45 \mathrm{GHz}$ ) is available, and it had been successfully used in the HL-1M tokamak for current drive [2]. To know the prospective operation scenario upon injection of higher power LH wave into the single-null divertor plasma, the effectiveness of plasma heating by electron Landau interaction is investigated with numerical simulation.

Significant progress has been achieved in reducing anomalous energy transport in tokamak plasmas. In addition to the well-known improved $\mathrm{H}$-mode regime with a transport barrier at the plasma edge, recent experiments performed in most tokamaks have shown that spontaneous reduction in anomalous transport can also occur inside the plasma core to form an internal transport barrier (ITB). Though the ion-ITB, which manifests itself by higher gradient of ion temperature inside it, has been studied extensively, the database and the physics understanding of electron-ITBs (eITB) are not so much extended as those of ion-ITB. In many tokamaks, such as FTU, DIII-D, TCV, ASDEX Upgrade, JT-60U and RTP [3-8], electron cyclotron resonance heating (ECRH) has been used to enter in improved confinement electron mode and eITBs have been observed. Most of the experiments were

*Address correspondence to this author at the Southwestern Institute of Physics, P O Box 432, Chengdu 610041, P.R. China;

E-mail: qgao@swip.ac.cn done in conditions of dominant electron heating in low collisional plasma. By using the available lower hybrid scheme in HL-2A, the plasma heating by electron Landau interaction can establish operation scenarios of preferentially dominant electron heating in low density plasmas.

The plasma transport through electron channel is larger than the neoclassical prediction by about two orders of magnitude, which is attributed to micro-turbulence. The electron transport is affected by turbulence of all wavelength, that is short wavelength $\left(k_{\theta} \rho_{s}>1\right.$, where $k_{\theta}$ is the poloidal wave vector and $\rho_{s}$ the ion gyro-radius calculated at $T_{e}=T_{i}$ ) like electron temperature gradient mode (ETG), intermediate wavelength $\left(k_{\theta} \rho_{s} \sim 1\right)$ like trapped electron mode (TEM), and long wavelength $\left(k_{\theta} \rho_{s} \sim 0.1\right)$ turbulence like ion temperature gradient mode (ITG). Gyrofluid simulation [9] and gyrokinetic simulation [10] have found the stabilizing effect of reversed magnetic shear (RS) and large pressure gradient in the $(\hat{s}-\alpha)$ ballooning diagram (where $\hat{s}$ is the magnetic shear, $\left.\alpha=-q^{2} R \beta^{\prime}\right)$ on the TEM/ETG instabilities. Consequently, control of the current profile is an important means to reduce anomalous transport in the electron channel. As the LH wave is of efficient current drive capability, it can be used to control the current profile to form the RS configuration. In the paper we will study the profile control under the dominant electron heating condition by using the tokamak discharge simulation.

\section{Flux Geometry of Single-Null Divertor Configuration}

A fast algorithm is elaborated for identifying the plasma boundary from measurements performed with the pick-up coils and magnetic flux loops. The algorithm is based on modeling the plasma current by movable current filaments, and it aims at being applied to real-time data processing (online mode). The algorithm was tested in a series of model discharges under conditions typical of the HL-2A tokamak. 
By using the algorithm, separatrix of the SND plasma was identified, and the determined striking area on the divertor target plate is in agreement with the measurements of electric probe array. As an example, the measured plasma boundary of shot $1776 \#$ at $t=0.53 \mathrm{~s}$ is shown in Fig. (1).

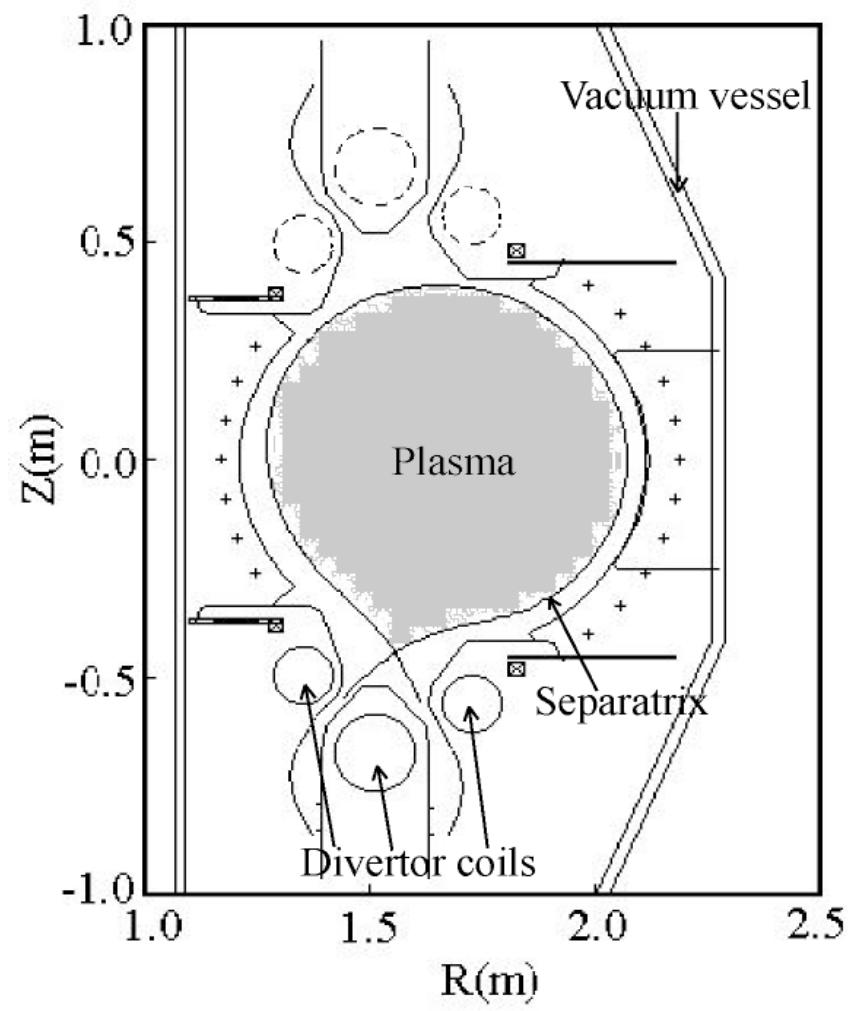

Fig. (1). Schematic of the cross-section of the HL-2A device, and separatrix of the single-null divertor plasma (shot 01766) identified by a fast algorithm. The plus signs "+" symbolize the pick-up coils.

In the simulated discharge, the plasma current ramps up to the maximum during $\sim 0.3$ second and then maintain a flat-top for about 1.6 second. The boundary (such as the $99.9 \%$ flux surface of the plasma) of the SND plasma that has been determined in the HL-2A discharge is used to describe the plasma geometry. The boundary is specified as a function of time, which evolves from a circular limiter plasma to a SND plasma such as that shown in Fig. (1) during the first $0.35 \mathrm{~s}$ and then retains the same boundary in the flat-top phase. The interior flux surfaces are computed by solving the Grad-Shafranov equation. The flux geometry is shown in Fig. (2) where the boundary surface is designated $\rho$ $=1$, where $\rho$ is defined as the square-root of normalized toroidal flux. The boundary surface is up-down asymmetric with different triangularity shaping for up-half plasma and low-half plasma. The lower triangularity is $\delta_{L}=0.25$, but the upper triangularity $\delta_{U} \sim 0$. Though the plasma has a mildly shaped boundary, the flux surfaces inside plasma are nearly circular.

\section{LH Wave Absorption by Landau Damping}

By choosing a local Cartesian coordinate system [11], such that $\hat{z} \times \mathbf{B}=0$ and the wave vector $\vec{k}$ is contained in the $\mathrm{x}-\mathrm{z}$ plane: $\vec{k}=k_{/ /} \hat{z}+k_{\perp} \hat{x}$,

the LH wave dispersion relation is decomposed into its real and imaginary parts,

$D=D_{r}+i D_{i}=0$

with

$$
\begin{aligned}
D_{r}= & -\alpha k_{\perp}^{6}+\varepsilon_{\perp} k_{\perp}^{4}+\left[\left(\varepsilon_{/ /}+\varepsilon_{\perp}\right)\left(k_{/ /}^{2}-\varepsilon_{\perp} k_{0}^{2}\right)+\varepsilon_{x y}^{2} k_{0}^{2}\right]+ \\
& \varepsilon_{/ /}\left[\left(k_{/ /}^{2}-\varepsilon_{\perp} k_{0}^{2}\right)^{2}-\varepsilon_{x y}^{2} k_{0}^{4}\right] \\
D_{i}= & \frac{\partial D_{r}}{\partial \varepsilon_{/ /}} K_{z z, i}
\end{aligned}
$$

where $k_{0}=\omega / c, \varepsilon_{/ /}, \varepsilon_{\perp}$, and $\varepsilon_{x y}$ are components of the dielectric tensor for a cold plasma, $\alpha$ is the thermal term that would be important near lower hybrid resonance,

$\alpha=\frac{3}{4} \frac{\omega_{p e}^{2}}{\omega_{c e}^{4}} v_{T e}^{2}+3 \frac{\omega_{p i}^{2} v_{T i}^{2}}{\omega^{4}}$

where $\omega_{p e}$ and $\omega_{p i}$ are the plasma frequencies, $v_{T e}$ and $v_{T i}$ are the thermal velocities. The anti-Hermitian part of the dielectric tensor $\vec{K}$ is retained as a perturbation. Here, the principal such term enters as an imaginary correction to $K_{z z}$, which describes the Landau damping of the LH wave in plasmas, namely the interaction between the wave electric field component parallel to $\vec{B}$ and electrons whose speed along $\vec{B}$ matches that of the wave:

$$
K_{z z, i}=-\pi \frac{\omega_{p e}^{2}}{\omega} \int d v_{/ /} v_{/ /} \frac{\partial f_{e}}{\partial v_{/ /}} \delta\left(\omega-k_{/ /} \nu_{/ /}\right)
$$

where $f_{e}\left(v_{/ l}\right)$ is a parallel velocity distribution function of electrons normalized such that $\int d v_{/ /} f_{e}\left(v_{/ /}\right)=1$.

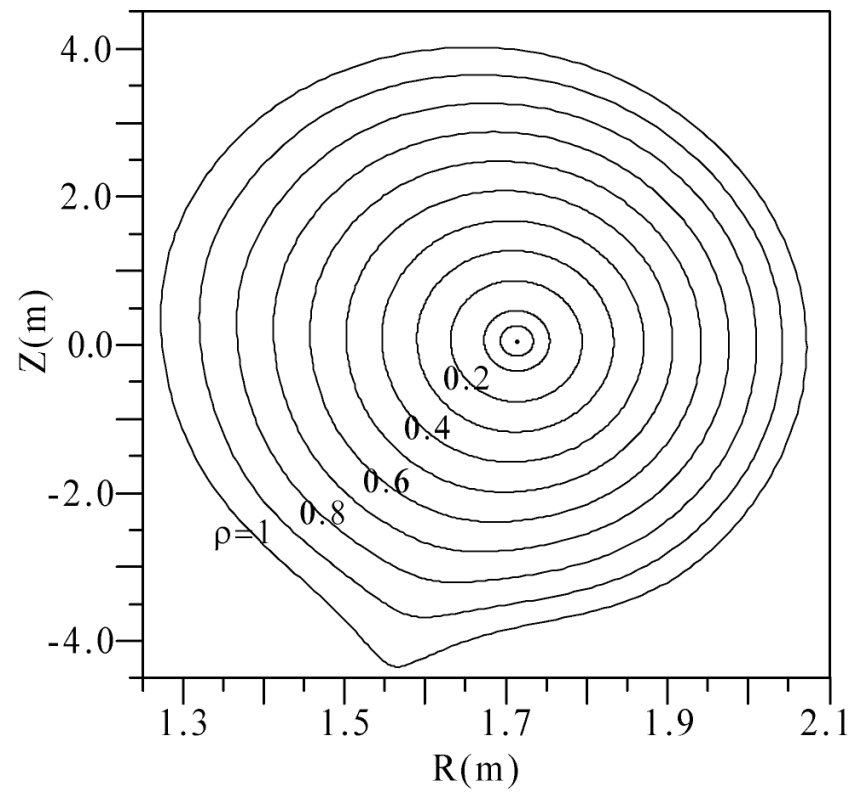

Fig. (2). Magnetic flux surfaces computed during LH heating with the plasma boundary defined by the separatrix shown in Fig. (1). 
The calculation for the LH wave absorption utilizes a toroidal ray-tracing algorithm for the wave propagation and a parallel velocity Fokker-Planck calculation for the interaction of waves and particles. Here a 1-D collision operator is used in the Fokker-Planck equation [12].

A spectral component of power $W$ experiences a change $\Delta W$ over time interval $\Delta \tau$ :

$$
\begin{aligned}
& \Delta W=-2 D_{i} /\left(\frac{\partial D_{r}}{\partial \omega}\right) W \Delta r=-2 \frac{\partial D_{r}}{\partial \varepsilon_{/ /}} K_{z z, i} /\left(\frac{\partial D_{r}}{\partial \omega}\right) W \Delta r \\
& =2 \pi \frac{\omega_{p e}^{2}}{\omega} \int d v_{/ /} v_{/ /} \delta\left(\omega-k_{/ /} v_{/ /}\right) \times \frac{\partial D_{r}}{\partial \varepsilon_{/ /}} /\left(\frac{\partial D_{r}}{\partial \omega}\right) W \Delta r
\end{aligned}
$$

The current driven on each flux surface is calculated according to

$$
j_{L H}=\frac{-e n_{e}}{v_{r}} \int d v_{/ /} D_{q l}\left(v_{/ /}\right) \frac{\partial f_{e}\left(v_{/ /}\right)}{\partial v_{/ /}} \frac{\partial W_{s}(u)}{\partial u}
$$

where

$$
D_{q l}\left(v_{/ /}\right)=\frac{\pi}{2}\left(\frac{e}{m_{e}}\right)^{2} E_{/ /}^{2} \delta\left(\omega-k_{/ /} v_{/ /}\right)
$$

$v_{r}=(\ln \Lambda) n_{e} e^{4} / 4 \pi \varepsilon_{0}^{2} m_{e}^{2} /\left|v_{r}\right|^{3}$,

$$
v_{r}=-\operatorname{sgn}\left(e E_{D C}\right) \sqrt{n_{e} e^{4} \ln \Lambda / 4 \pi \varepsilon_{0}^{2} m_{e}\left|e E_{D c}\right|} .
$$

The key quantity is $W_{s}(u)$, the energy (normalized to $m_{e} v_{r}^{2} / 2$ ) imparted to the electric field $E_{D C}$ by an electron as it slows down.

\section{Lower Hybrid Wave Heating and eITB}

The LH wave is injected with the HL-2A multi-junction launcher $(2 \times 12)$. The radiated power spectrum by the launcher is calculated with the Brambilla coupling theory [13]. The radiated power spectrums are controlled by the relative wave-guide phasing $(\Delta \varphi)$ in the wave-guide array. Some spectrums radiated by the launcher are calculated as shown in Fig. (3). The asymmetric power spectrum $(\Delta \varphi=$ $90^{\circ}$ ) is used for current drive, and the symmetric spectrum $\left(\Delta \varphi=180^{\circ}\right)$ is used for plasma heating. In order to produce some non-inductive current to control the current profile, a nearly symmetric spectrum of $\Delta \varphi=170^{\circ}$ is assumed in the LH heating.

TRANSP is a plasma analysis code used to model the time evolution of plasma parameters in tokamak discharges [14]. The Brambilla coupling calculation combined with the LH absorption calculation is in conjunction with the TRANSP code to obtain the plasma heating in a dynamic case. The local electric field $E_{D C}$ is supplied by TRANSP as part for iteration.

The energy transport model used in the simulation is a mixed theory model. Normally the transport observed in tokamak experiments greatly exceeds that of collisional transport theory. However, no theoretical model has yet been proposed which adequately describes all of the many features displayed by tokamak transport. Here, the ion heat diffusivity is assumed in terms of neoclassical transport enhanced by $\eta_{i}$ turbulence. The electron energy transport is based on the Rebut-Lallia-Watkins (RLW) model [15] which, from heuristic and dimensional arguments, introduces a critical electron temperature gradient $\nabla T_{e c}$ such that the electron heat flow is neoclassical when $\left|\nabla T_{e}\right|<\left|\nabla T_{e c}\right|$ or $\nabla q<0$. The distinctive feature of this hybrid model is that both $\eta_{i}$ model and RLW model have been tested against a wide range tokamak devices [16].

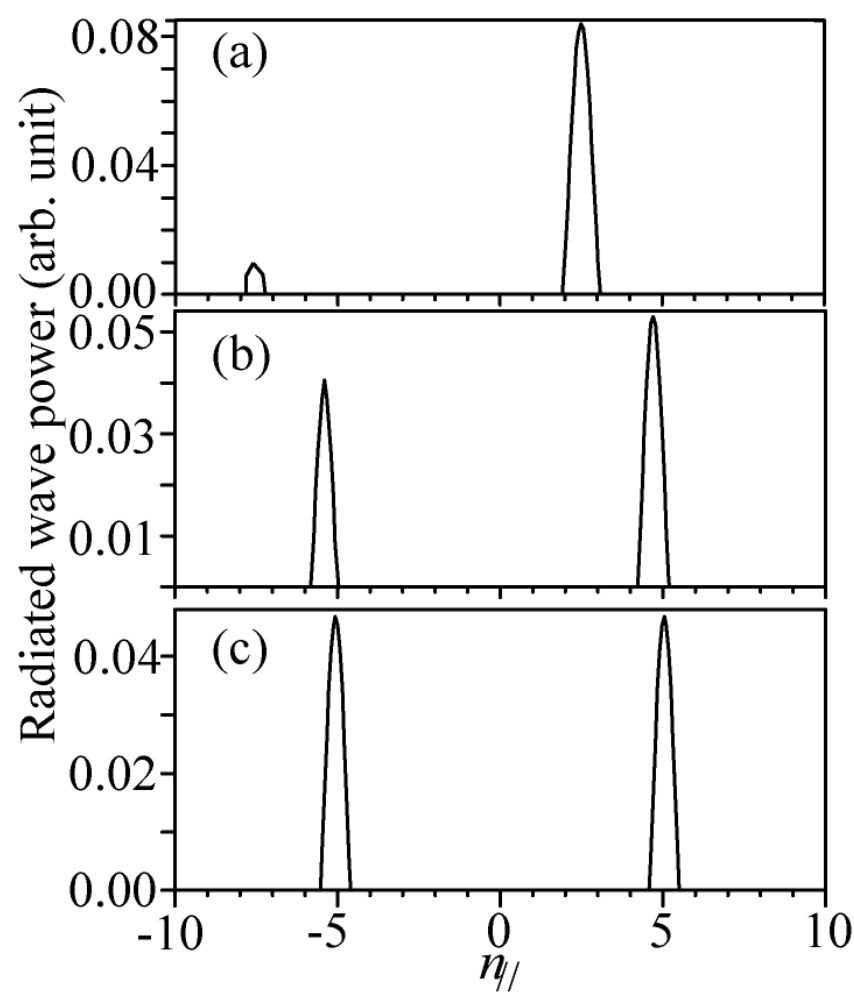

Fig. (3). Radiated LH wave power versus $n_{/ /}$. (a) $\Delta \varphi=90^{\circ}$, (b) $\Delta \varphi$ $=170^{\circ}$, (c) $\Delta \varphi=180^{\circ}$.

Scenario of the low-density plasma heated by higher LH power is investigated. The parameters of the target plasma are: $I_{p}=220 \mathrm{kA}, B_{T}=2.0 \mathrm{~T}, \quad$ and $\quad \bar{n}_{e}=1.0 \times 10^{19} \mathrm{~m}^{-3}$, deuterium gas. $1.5 \mathrm{MW}$ LH wave power is injected during the flat-top phase $(\mathrm{t}=0.7-1.2 \mathrm{~s})$. Due to higher power electron Landau heating, operation scenarios of preferentially dominant electron heating is established in low density plasma. The electron temperature increases significantly during LH heating, namely the central temperature rises to more than $1.4 \mathrm{keV}$ from about $0.6 \mathrm{keV}$ (Fig. 4). In contrast to the large increment of the electron temperature, the ion temperature only has a small change (namely, the central $T_{i}$ rise $\Delta T_{i 0} \approx 0.17 \mathrm{keV}$ from the Ohmic value $T_{i 0}=0.5 \mathrm{keV}$ ) because of low plasma density.

Since the parallel refractive index of the used LH power spectrum $\left(\Delta \varphi=170^{\circ}\right)$ is rather high (the central refractive index $n_{/ / 0} \approx 5.0$ ), the injected LH wave with lower phase velocity can be absorbed in outer region resulting in off-axis electron heating (Fig. 5a). The analysis of wave deposition 
region in the $\left(\rho, n_{/ /}\right)$phase space shows that constraint imposed by the wave propagation condition limits the maximum allowed $n_{/ /}$-upshift [17]. Taking into account the Landau damping condition, it is shown that LH power deposits off-axis. In addition to the plasma heating, a noninductive current $\left(I_{L H} \approx 80 \mathrm{kA}\right.$ ) is driven by the LH wave because of the slight asymmetry of the LH wave spectrum and the asymmetry of electron velocity distribution function caused by the electric field, $E_{D C}$. Due to the off-axis driven current, RS q-profile, of which the magnetic shear is weak in the central region and negative in the mid-plasma region $(\rho \approx$ $0.45-0.65$ ), is formed (Fig. 5b).

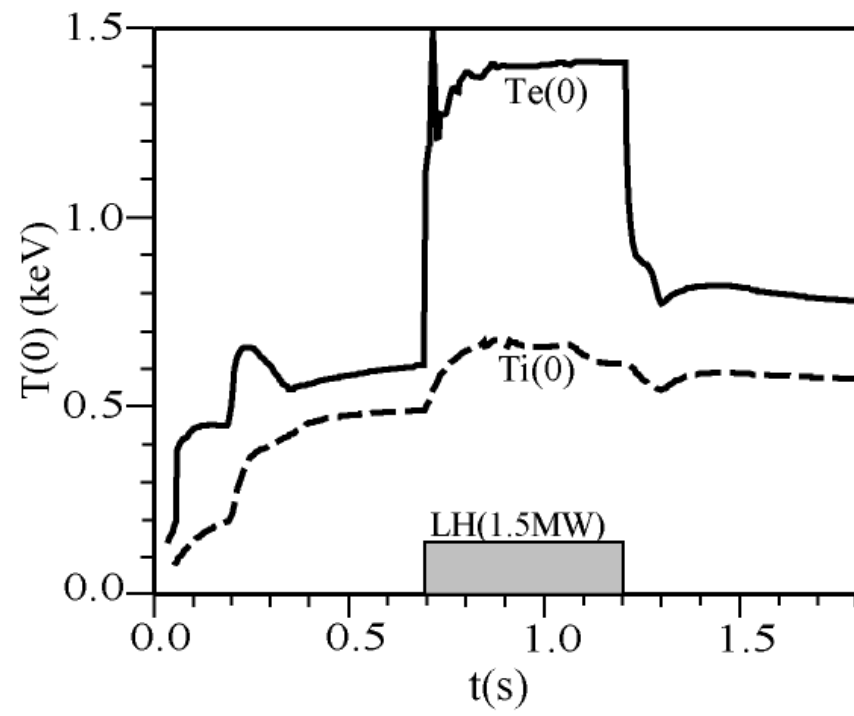

Fig. (4). Temporal evolution of the central electron temperature (full line) and the central ion temperature (dashed line).

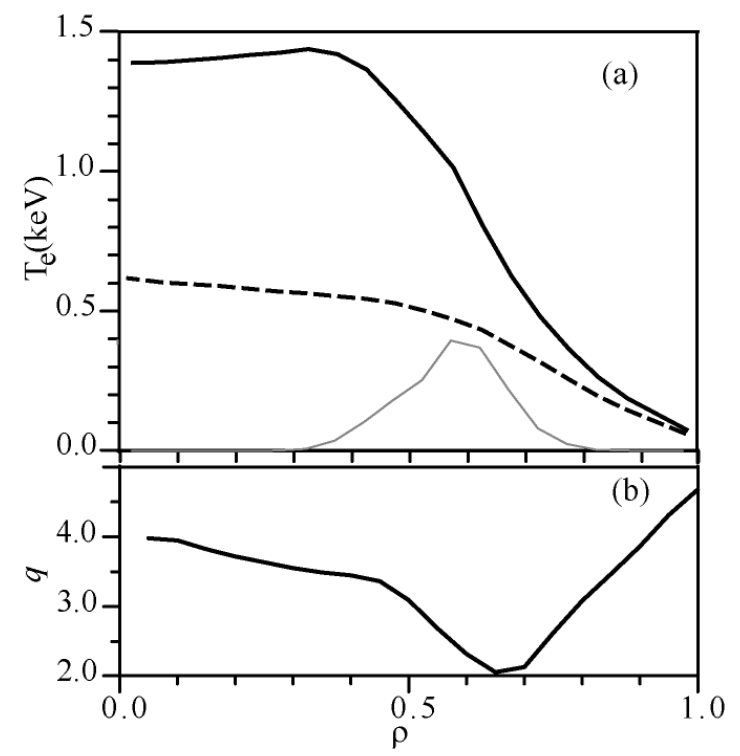

Fig. (5). (a) Electron temperature profiles during LH heating phase at $\mathrm{t}=0.9 \mathrm{~s}$ (full line), and during Ohmic heating only at $\mathrm{t}=0.68 \mathrm{~s}$ (dashed line). Fainter line indicates the location of the LH power absorption. (b) Profile of $q$ at $\mathrm{t}=0.9 \mathrm{~s}$ during LH heating.

The profiles of electron temperature during the Ohmic heating and LH heting phase are plotted in Fig. (5a). It is shown that LH heating raises electron temperature largely and a steep temperature gradient is formed around the magnetic shear reversal region. The normalized temperature gradient at the steepest gradient, where $\rho=0.65$ and $T_{e}=$ $0.75 \mathrm{keV}$, is $R / L_{T}=18$ (where $L_{T}^{-1}=\nabla T_{e} / T_{e}$ ). This gradient exceeds largely the critical gradient value $\left(R / L_{T}<10\right)$ for temperature profile stiffness [18]. In normal tokamak configuration, the critical gradient is thought to be the threshold to drive short or intermediate wavelength ETG/TEM instabilities leading to 'stiff' transport behavior. The large normalized temperature gradient produced in the profile controlled discharge is consistent with the expected stabilizing effect of weak/negative shear on ETG/TEM instabilities. In FTU and DIII-D [3, 4], ECRH has been used during fast current ramps to obtain negative or flat q-profile by using the skin effect. In the FTU experiment the eITB was developed with extremely high temperature gradient. The maximum normalized gradient of $R / L_{T}=19$ was obtained, implying that the plasma behaves as it is far from the stiff critical gradient. In DIII-D, eITB was established with injecting ECRH off-axis into a low-density plasma, and an extreme steep temperature gradient was formed outside the power deposition region. The gyrokinetic linear stability analysis on DIII-D showed that the experimentally measured $\nabla T_{e}$ at the barrier was very close to the expected critical gradient for ETG mode, provided the stabilizing effect of negative shear and large pressure gradient in the $(\hat{s}-\alpha)$ ballooning diagram was included in the calculation to reduce the turbulence growth rate.

As in the case of ion-ITB one would clarify an eITB when a clear decrease of the electron thermal conductivity is observed at the steep temperature gradient or in the region inside it. The simulation results show that the electron thermal conductivity is reduced obviously at the steep temperature gradient and in the central plasma region (Fig. 6). GKF transport model is a first-principle model of anomalous transport based on numerical simulation, with stringent comparisons to experimental data from the TFTR tokamak [19]. This model is based on nonlinear gyrofluid simulations, which predict the fluctuation and thermal transport characteristics of toroidal ion-temperature-gradientdriven (ITG) turbulence, and on comprehensive linear gyrokinetic ballooning calculations, which provide very accurate growth rates, critical temperature gradients, and a quasilinear estimate of $\chi_{e} / \chi_{i}$ (where $\chi_{e}$ and $\chi_{i}$ are the electron and ion thermal conductivity respectively). By adding the anomalous transport resulted from the GKF model to the neoclassical transport, the evaluation of electron thermal conductivity shows that it decreases obviously at the steep temperature gradient (Fig. 6), which is of similar trend to the electron heat diffusivity of the simulated plasma.

A parameter used to describe the eITB characteristics in JET is [20]

$\rho_{T}^{*}(R, t)=\rho_{s}(R, t) / L_{T e}(R, t)$

where $L_{T e}=-T_{e} /\left(\partial T_{e} / \partial R\right)$ is the local temperature gradient scale length, $R$ the plasma major radius on the equatorial plane, $\rho_{s}=c_{s} / \omega_{c i}$ the ion Larmor radius at the sound speed, $c_{s}$ 
the ion sound speed and $\omega_{c i}$ is the ion gyro-frequency. The studies of turbulence transport through extensive use of computer code have found that a possible mechanism for the stabilization of ITG modes and TEMs in tokamaks combines the $\vec{E} \times \vec{B}$ rotation flow and the magnetic shear effects. The dimensional analysis of the stabilization criterion $\gamma_{E \times B} \geq \gamma_{\max }$ (where $\gamma_{E \times B}$ is the $\vec{E} \times \vec{B}$ shearing rate, and $\gamma_{\max }$ is the maximum linear growth rate of ITG modes) shows that the strength of the eITB is quantified by the maximum value of $\rho_{T}^{*}(R, t)$ profile and its location. In the TCV tokamak, the eITB has been measured by the location of the maximum $\rho_{T}^{*}$ to illustrate the direct relationship between the current profile and the eITB development. In $\mathrm{TCV}$, strongly reversed magnetic shear configuration was formed by using electron cyclotron current drive (ECCD), and a well-established eITB was developed consistently. As the Ohmic current penetrates through the plasma minor radius and results in a strongly peaked current density contribution, the strong reversed magnetic shear decreases or eliminates. Consequently, the eITB decreases in strength and in radius as measured by the maximum value of $\rho_{T}^{*}(R, t)$ profile and its location [21]. Here in the simulated HL-2A discharge, the profile of $\rho_{T}^{*}$ shows that the maximum value of $\rho_{T}^{*}(R, t)\left(\rho_{\text {Thax }}^{*} \approx 0.022\right)$ is located around the shear reversal point (Fig. 7), implying that the eITB is developed because of the magnetic shear reversal.

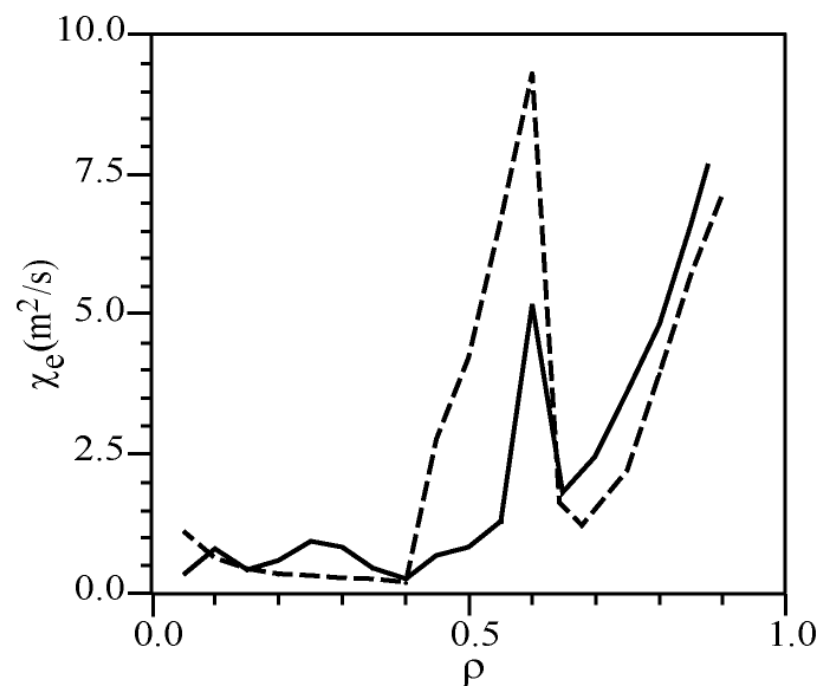

Fig. (6). Electron thermal conductivity (full line), and electron thermal conductivity evaluated from the GKF model (dashed line) versus $\rho$.

To realize both plasma heating and current profile control by using LH wave, an alternative way is that the LH wave is injected with double-antenna: one antenna is used to radiate symmetric spectrum $\left(\Delta \varphi=180^{\circ}\right)$ for heating, and another radiates asymmetric spectrum $\left(\Delta \varphi=90^{\circ}\right)$ for current drive. By adjusting the LH power for current drive, a discharge with similar features as in the single-antenna case could be obtained. With $1.5 \mathrm{MW} \mathrm{LH}$ power used for heating and $0.13 \mathrm{MW}$ for LHCD, the electron temperature profile and $q$ profile similar to the profiles shown in Fig. (5) are produced
(Fig. 8). The results demonstrate that the effects of plasma heating and current profile control achieved by a single antenna of appropriate radiated spectrum are equivalent to that produced with LH wave injected by double-antenna.

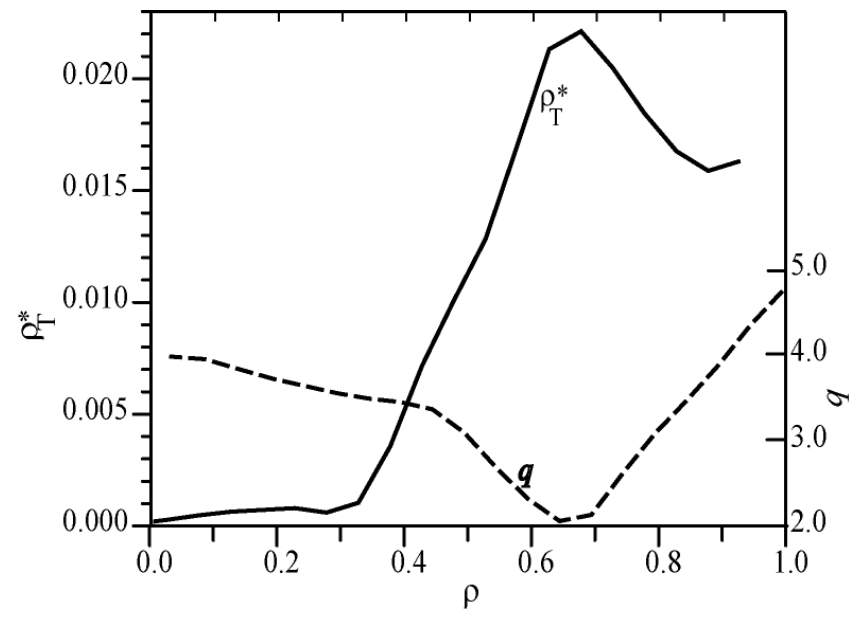

Fig. (7). Profile of $\rho_{T}^{*}$ (full line) and profile of $q$ (dashed line).

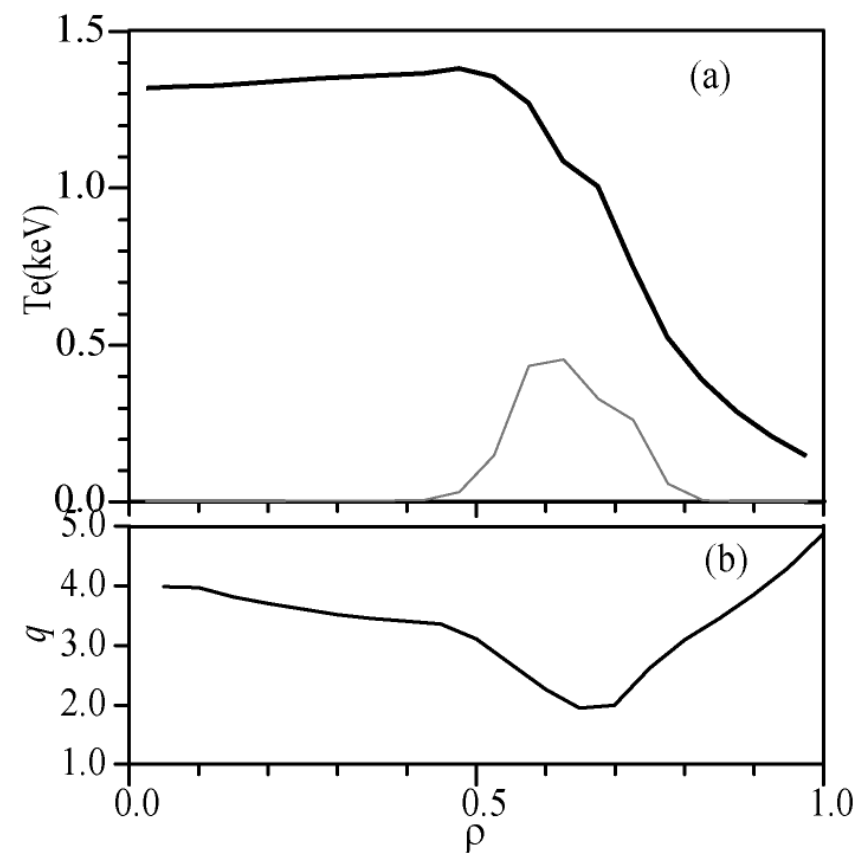

Fig. (8). (a) Profile of electron temperature. Fainter line indicates the location of LH absorption. (b) Profile of $q$.

\section{Enhanced Ion Confinement}

A neutral beam injection (NBI) line of lower energy has been developed and used for perpendicular injection in the HL-1M tokamak [22]. To raise the parameters of the target plasma for LH heating in HL-2A, the NBI line is used to heat the low-density plasma. The low-density plasma as described in above section is heated by co-injected NBI $\left(P_{N B}\right.$ $=0.5 \mathrm{MW})$ during $0.5-1.8 \mathrm{~s}$, and then a higher $\mathrm{LH}$ power $\left(P_{L H}\right.$ $=1.5 \mathrm{MW}$ ) is injected (with the slightly asymmetric spectrum of $\Delta \varphi=170^{\circ}$ ) during 1.0-1.5s to heat the plasma further and keep the $q$-profile reversed. During the LH wave injection phase, not only the electron temperature has a large increment, but the ion temperature increases significantly (from $T_{i 0}=1.5 \mathrm{keV}$ to $T_{i 0} \sim 2.6 \mathrm{keV}$ ) as well (Fig. 9). As 

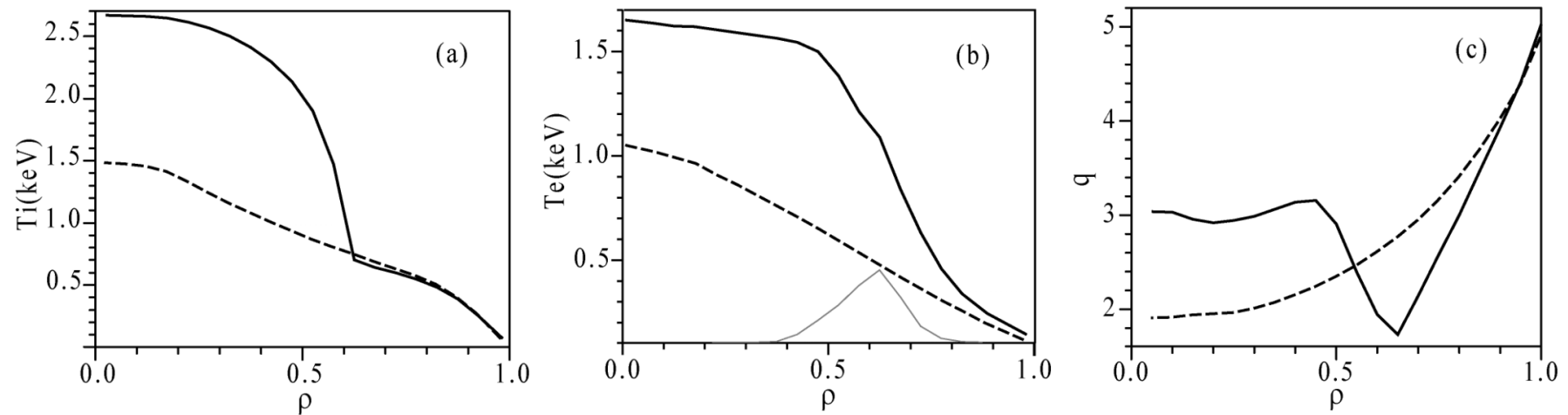

Fig. (9). (a) Profiles of ion temperature, (b) Profiles of electron temperature (fainter line indicates the location of LH wave deposition), and (c) Profiles of q. Dashed lines correspond to the case of NBI heating only at $\mathrm{t}=0.9 \mathrm{~s}$, and full lines correspond to the case of NBI+LH heating at $\mathrm{t}=1.2 \mathrm{~s}$.

indicated in above section, the ion temperature only has a small increment when the Ohmic target plasma is heated by the LH wave. Nevertheless, with the same LH wave injected into the NBI heated target plasma of higher temperature, the ion temperature increases largely implying that the plasma enters in enhanced confinement ion mode. As shown in Fig. (9), the temperature profiles indicate that an enhanced core confinement regime is established with both ion-ITB and eITB in the LH heating phase.

The beam power for heating and the beam power losses during the NBI heating only phase and that during the NBI + LH heating phase are listed in Table $\mathbf{1 .}$

The interaction of beam with plasma predicts that the increase of $T_{e}$ caused by the LH heating should increase the rate of NBI energy going into the bulk ions. Nevertheless, we see, from table 1 , that when the electron temperature increases during LH heating, the fraction of NBI power going into the bulk ions is not increased (actually, it is decreased a bit), while that going into the electrons is reduced obviously as expected. The reason causing this inconsistency is that both the power from the beam ions thermalization and the beam power loss caused mainly by charge-exchange increase. The NBI power that goes directly into the bulk ions and the power introduced by thermalization of the beam ions compose the ion heating power, which is nearly unchanged (see table 1). For low density plasma, the effect of electron-ion equipartition is negligible. Thus, compared to the case of NBI heating only, the large increment of $T_{i}$ within the transport barrier should be attributed to the reduction of ion heat transport resulted from the optimizing magnetic shear.

To illustrate the positive effects of the RS configuration on the ion confinement, a comparison for the ion confinement is made between the RS and non-RS plasma. In the above NBI heated plasma, an optimized q-profile with negative shear is produced when the LH wave is injected with slightly asymmetric spectrum $\left(\Delta \varphi=170^{\circ}\right)$, and it is referred to RS plasma. Alternatively, when the LH wave is injected with purely symmetric spectrum $\left(\Delta \varphi=180^{\circ}\right)$, the $q$ profile with negative shear could not be formed (Fig. 10) because the off-axis current driven by the LH wave is not sufficient. It is referred to non-RS plasma. Comparing the plasma performance in the profile controlling phase $(\mathrm{t}=1.0$ $1.5 \mathrm{~s})$, we see that in the non-RS plasma, only much lower ion temperature $\left(T_{i 0} \approx 2.0 \mathrm{keV}\right)$ is obtained (Fig. 11b), and the ion energy confinement time is reduced by $20-25 \%$ with respect to that in the RS case (Fig. 11a).

\section{CONCLUSIONS}

Lower hybrid wave is injected into a single-null divertor plasma in HL-2A. Dominant electron heating and current profile control are investigated with numerical simulation. The magnetic flux surfaces are calculated by solving the Grad-Shafranov equation using the SND plasma boundary that is determined from the HL-2A discharge by a filament model. The LH wave power spectrum radiated by a multijunction launcher $(2 \times 12)$ is calculated with the Brambilla's coupling theory. Analysis of the LH wave absorption utilizes a toroidal ray-tracing for the wave propagation and a parallel velocity Fokker-Planck calculation for the interaction of waves and particles. The LH absorption calculation combined with the Brambilla coupling calculation is in conjunction with the TRANSP code to obtain the plasma heating in a dynamic case.

Scenario of the low-density plasma heated by higher LH power is investigated. Plasma heating by electron Landau interaction results in operation scenarios of preferentially dominant electron heating. When LH wave $\left(P_{L H}=1.5 \mathrm{MW}\right)$ is injected to a low-density plasma with relative wave-guide phase $\Delta \varphi=170^{\circ}$, a non-inductive current $\left(I_{L H} \approx 80 \mathrm{kA}\right)$ is

Table 1. The NBI Heating Power and the Beam Power Losses ${ }^{1}$

\begin{tabular}{|c|c|c|c|c|c|c|c|}
\hline & $\mathbf{P}_{\text {bi }}(\mathbf{k W})$ & $\mathbf{P}_{\text {be }}(\mathbf{k W})$ & $\mathbf{P}_{\text {bth }}(\mathbf{k W})$ & $\mathbf{P}_{\text {cx }}(\mathbf{k W})$ & $\mathbf{P}_{\text {shin }}(\mathbf{k W})$ & $\mathbf{P}_{\text {orb }}(\mathbf{k W})$ & $\mathbf{P}_{\text {ie }}(\mathbf{k W})$ \\
\hline \hline NBI phase $(\mathrm{t}=0.95 \mathrm{~s})$ & 202.4 & 116.9 & 30.7 & 116.9 & 24.8 & $\sim 5.0$ & -17.6 \\
\hline NBI+LH phase $(\mathrm{t}=1.2 \mathrm{~s})$ & 187.4 & 53.1 & 60.2 & 156.2 & 31.7 & $\sim 8.0$ & -14.5 \\
\hline
\end{tabular}

${ }^{1} \mathrm{P}_{\mathrm{bi}}$ is the NBI power that goes into ions, $\mathrm{P}_{\mathrm{be}}$ the NBI power that goes into electrons, $\mathrm{P}_{\mathrm{bth}}$ the power from thermalization of the beam ions, $\mathrm{P}_{\mathrm{cx}}$ the NBI power lost by charge-exchange, $\mathrm{P}_{\text {shin }}$ the NBI power shone through, $\mathrm{P}_{\text {orb }}$ the orbit loss power of the beam ions, and $\mathrm{P}_{\mathrm{ie}}$ the power loss by electron-ion coupling inside the ion-ITB. 
driven in addition to the plasma heating. Due to the off-axis driven current, RS q-profile is formed under the dominant electron heating condition. The normalized electron temperature gradient around the shear reversal exceeds the critical value largely. This implies that short or intermediate wavelength ETG/TEM instabilities, which cause resilience of the electron temperature profile, are suppressed due to the stabilizing effect of reversed magnetic shear. The clear decrease of electron thermal conductivity and the location of maximum $\rho_{T}^{*}$ indicate that an eITB is developed in the vicinity of the shear reversal. Furthermore, the effects of plasma heating and current profile control obtained by asymmetric LH spectrum in some sort $\left(\Delta \varphi=170^{\circ}\right)$ are equivalent to that obtained by using double-antenna: one antenna radiating symmetric spectrum $\left(\Delta \varphi=180^{\circ}\right)$ for heating, and another radiating completely asymmetric spectrum $\left(\Delta \varphi=90^{\circ}\right)$ for current drive.

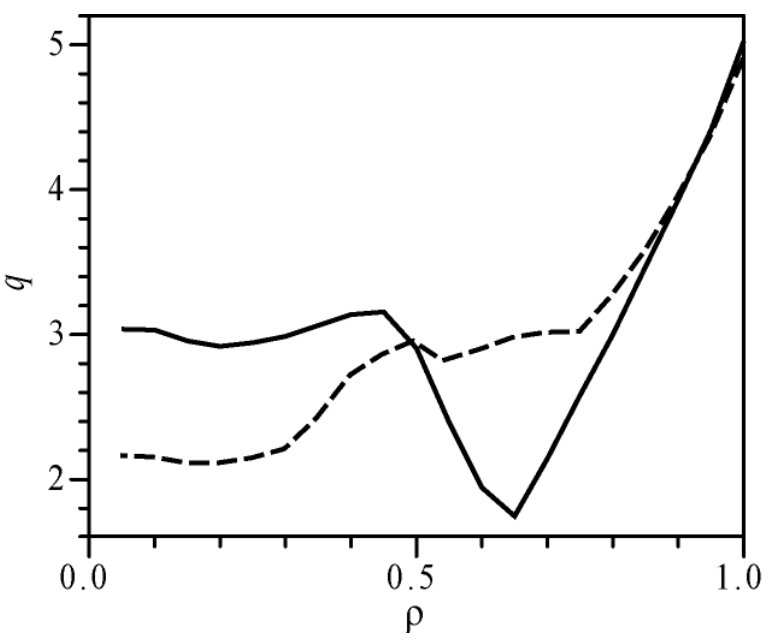

Fig. (10). Profile of $q$ for the case of RS plasma (full line), and the case of non-RS plasma (dashed line).

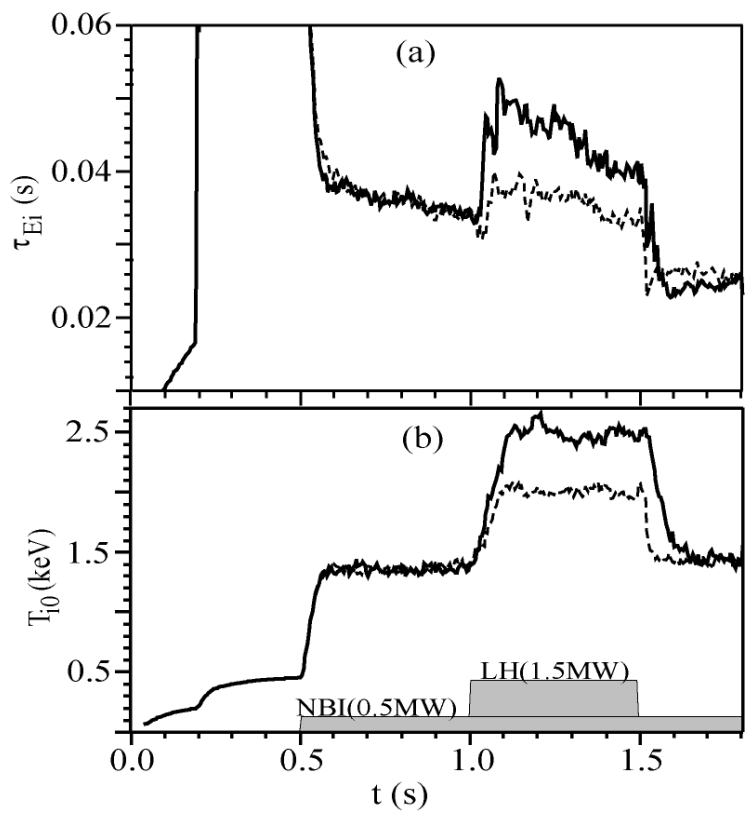

Fig. (11). Temporary evolution of (a) the ion energy confinement time, and (b) the central ion temperature. Full lines correspond to the case of RS plasma, and dashed lines correspond to the case of non-RS plasma.
The simulation of LH heating in the target plasma heated by NBI shows that the modification of current profile caused by the $\mathrm{LH}$ driven current is favorable to enhancing the ion energy confinement. As higher LH power injected into the NBI heated plasma with slightly asymmetric spectrum $(\Delta \varphi=$ $170^{\circ}$ ), the ion temperature has a large increment in addition to the high increase of electron temperature. The temperature profiles indicate that an enhanced core confinement is established with both ion-ITB and electron-ITB developed. Nevertheless, when the same LH power is injected into the NBI heated plasma with purely symmetric spectrum $(\Delta \varphi=$ $180^{\circ}$ ), RS configuration can not be established due to not enough off-axis current driven by the LH wave. Consequently, only much lower ion temperature can be obtained, and the ion energy confinement time is reduced by $20-25 \%$ comparing to the RS plasma configuration.

\section{ACKNOWLEDGEMENTS}

This work is supported by the National Natural Science Foundation of China under Grant No 10275018. We are grateful to R. V. Budny, D. C. McCune and TRANSP team at PPPL for facilitating the TRANSP operation.

\section{REFERENCES}

[1] Gao QD, Budny RV, Zhang JH, Li FZ, Jiao YM. Sustained reversed magnetic shear mode of operation in the HL-2A tokamak. Nucl Fusion 2000; 40: 1897-907.

[2] Liu Y, Li XD, Wang EY, et al. Confinement studies during LHCD and LHW ion heating on HL-1M, in Fusion Energy 1998 (17th Int Conf. Yokohama), Conference proceedings, IAEA, Eds. 1999; EXP2/17. http://www-pub.iaea.org/MTCD/publications/PDF/csp 008c/fec1998/html/node159.htm.

[3] Buratti P, Barbato E, Bracco G, et al. High core electron confinement regimes in FTU plasmas with low- or reversedmagnetic shear and high power density electron- cyclotronresonance heating. Phys Rev Lett 1999; 82: 560-3.

[4] Prater R, Austin ME, Bernabei S, et al. Highly localized electron cyclotron heating and current drive and improved core transport in DIII-D, in Fusion Energy 2000 (18th Int. Conf. Sorrento), Conference proceedings, IAEA, Eds. 2001; EX8/1 http://wwwpub.iaea.org/MTCD/publications/PDF/csp_008c/html/node114.htm

[5] Pietrzyk ZA, Angioni C, Behn R, et al. Long-pulse improved central electron confinement in the TCV tokamak with electron cyclotron heating and current drive. Phys Rev Lett 2001; 86: 1530-

[6] Wolf RC, Günter S, Leuterer F, et al. Response of internal transport barriers to central electron heating and current drive on ASDEX Upgrade. Phys Plasmas 2000; 7: 1839-44.

[7] Ikeda Y, Ide S, Suzuki T, et al. ECRF experiments for local heating and current drive in JT-60U, in Fusion Energy 2000 (18th Int. Conf. Sorrento), Conference proceedings, IAEA, Eds. 2001; EXP4/03 http://www-pub.iaea.org/MTCD/publications/PDF/csp 008c/html/node $173 . \mathrm{htm}$.

[8] Lopes Cardozo NJ, Hogeweij G MD, Baar MDE, et al. Electron thermal transport in RTP: filaments, barriers and bifurcations. Plasma Phys Control Fusion 1997; 39: B303-16.

[9] Beer MA, Hammett GW, Rewoldt G, Synakowski EJ, Zarnstorff MC. Gyrofluid simulations of turbulence suppression in reversedshear experiments on the Tokamak Fusion Test Reactor. Phys Plasmas 1997; 4: 1792-9.

[10] Jenko F, Dorland W, Kotschenrenther M, Rogers BN. Electron temperature gradient driven turbulence. Phys Plasmas 2000; 7: 1904-10.

[11] Stix TH. Waves in Plasmas. New York : American Institute of Physics 1992.

[12] Ignat DW, Valeo EJ, Jardin SC. Dynamic modelling of lower hybrid current drive. Nucl Fusion 1994; 34: 837-51

[13] Brambilla M. Slow-wave launching at the lower hybrid frequency using a phased waveguide array. Nucl Fusion 1976; 16: 47-54. 
[14] Budny RV, Bell MG, Biglari H, et al. Simulations of deuteriumtritium experiments in TFTR. Nucl Fusion 1992; 32: 429-47.

[15] Rebut P-H, Watkins ML, Gambier DJ, Boucher D. A program toward a fusion reactor. Phys Fluids B 1991; 3: 2209-19.

[16] Conner JW. Transport in tokamaks: Theoretical models and comparison with experimental results. Plasma Phys Control Fusion 1995; 37: A119-34.

[17] Gao QD, Budny RV, Li FZ, Zhang JH. Predictive study of high performance scenarios in HL-2A. Nucl Fusion 2003; 43: 982-8.

[18] Ryter F, Angioni C, Beurskens M, et al. Experimental studies of electron transport. Plasma Phys Control Fusion 2001; 43: A323-38.

[19] Kotschenreuther M, Dorland W, Beer MA, Hammett GW. Quantitative predictions of tokamak energy confinement from first- principles simulations with kinetic effects. Phys Plasmas 1995; 2: 2381-9.

[20] Tresset G, Litaudon X, Moreau D, Garbet X, et al. A dimensionless criterion for characterizing internal transport barriers in JET. Nucl Fusion 2002; 42: 520-6.

[21] Martin YR, Henderson MA, Alberti S, et al. Accessibility and properties of ELMy H-mode and ITB plasmas in TCV. Plasma Phys Control Fusion 2003; 45: A351-66.

[22] Yan LW, Lei GJ, Zhong GW, et al. Neutral beam injection experiments in the HL-1M tokamak. Chin Phys Lett 2003; 20: $1795-8$.

(C) Gao et al.; Licensee Bentham Open.

This is an open access article licensed under the terms of the Creative Commons Attribution Non-Commercial License (http://creativecommons.org/licenses/by$\mathrm{nc} / 3.0 /$ ) which permits unrestricted, non-commercial use, distribution and reproduction in any medium, provided the work is properly cited. 